\title{
Analysis on the Impacts of Slant Tropospheric Delays on Precise Point Positioning
}

\author{
Chunbao Xiong ${ }^{1}$, Lina $\mathrm{Yu}^{1, *(1)}$ and Lewen Zhao ${ }^{2}$ \\ 1 School of Civil Engineering, Tianjin University, Tianjin 300072, China; luhai_tj@126.com \\ 2 School of Remote Sensing \& Geomatics Engineering, Nanjing University of Information Science \& Technology, \\ Nanjing 210044, China; lewen.zhao@pecny.cz \\ * Correspondence: yulina@tju.edu.cn
}

Received: 21 October 2019; Accepted: 9 November 2019; Published: 14 November 2019

Featured Application: The improvement of precise point positioning (PPP) accuracy can provide more wide application of high-accuracy PPP products on weather forecasts, structural healthy monitoring, etc.

\begin{abstract}
Tropospheric delay is one main factor affecting the accuracy of precise point positioning (PPP) ambiguity-float and fixed solutions. Investigations mainly focused on evaluating the contributions of tropospheric corrections to the accuracy and reliability of PPP solutions. The tropospheric corrections generally contained the zenith tropospheric delay (ZTD) and the horizontal gradients estimated from relative positioning or PPP. However, the estimated tropospheric delays can be partly absorbed by the carrier phase residuals if the stochastic model is not well-defined. Therefore, along with the ZTD and horizontal gradients, the carrier phase residuals from PPP backward filter are considered to reconstruct slant tropospheric delay (STD). Based on the proposed STD model, its marginal effects on GPS PPP were investigated. Results indicated that the consideration of carrier phase residuals for STD modeling can improve the three-dimensional accuracy to $0.5 \mathrm{~cm} / 1 \mathrm{~cm} / 1.2 \mathrm{~cm}$ in the South/North/Up $(\mathrm{N} / \mathrm{E} / \mathrm{U})$ components. Then, the effects of internal and external STD corrections on PPP float and fixed solutions were analyzed. Compared to the ZTD + gradients augmentation, STD corrections from the same station could improve the PPP accuracy by $51 \% / 51 \% / 60 \%$; the large improvements were because the multipath error and observation noise were eliminated. In comparison, the improvement was $14 \% / 28 \% / 31 \%$ using external STD corrections, which indicated the effects of unmodeled tropospheric errors in the phase residuals. The ambiguity-fixing results indicated that the fixing rate of PPP ambiguity was increased by $30 \%$ with STD augmentation. As the BeiDou System (BDS) suffered longer convergence than that of GPS, the benefits of STD modeling to the BDS observations were also validated. Overall, the results validated the performance of STD-augmented PPP, which demonstrated the potential application of high-accuracy troposphere products.
\end{abstract}

Keywords: precise point positioning; zenith tropospheric delay; slant tropospheric delay; carrier phase residuals

\section{Introduction}

Tropospheric delay is the main error source in Global Navigation Satellite System (GNSS) positioning and it is closely correlated with the positioning accuracy along up direction. Shi et al. [1] illustrated that the zenith tropospheric delay (ZTD) product provided by International GNSS Service (IGS) can improve the positioning accuracy of both float and fixed solutions. In addition, tropospheric delay based on ground-based GNSS is an important data source in meteorology, which can be used to determine the distribution of atmospheric water vapor, and used for short-term weather and rainstorm 
disaster forecast [2]. Different from the relative positioning, we can obtain high-accuracy un-differenced tropospheric delay using the precise point positioning (PPP) technique with high efficiency. It has been demonstrated to be a promising technology in real-time positioning and meteorological applications [3].

In traditional PPP data processing, the initial tropospheric delay is first calculated by an empirical model, then the residual tropospheric delay is used as an estimated parameter. There are mainly three methods for acquiring initial tropospheric value: (1) the traditional empirical tropospheric correction model; (2) the numerical weather model (NWM)-based empirical tropospheric model; (3) the real-time NWM tropospheric model. The empirical tropospheric correction model and NWM tropospheric model can reflect the seasonal variation of the troposphere. However, it cannot reflect the spatial and temporal characteristics of the troposphere in the short term, especially under extreme meteorological conditions. Therefore, scholars proposed applying the real-time NWM tropospheric model for PPP research. Lu et al. [4] proposed a four-system PPP method based on the NWM model; the experimental results showed that the tropospheric parameters obtained by NWM can improve the convergence times in the N/E/U direction by $20 \% / 32 \% / 25 \%$, respectively, and the corresponding positioning accuracy can be improved by $2.5 \% / 12.1 \% / 18.7 \%$. Lu et al. [5] obtained zenith tropospheric delay (ZTD) and horizontal gradients from the NWM model to enhance BeiDou PPP; the results showed that the convergence times in the east and up direction were increased by $60 \%$ and $66.7 \%$, respectively. Zhu et al. [6] proposed BeiDou and BeiDou/GPS-combined PPP based on the NWMmodel, and the results show that the convergence time of a single BeiDou PPP can be decreased by $80.6 \%$, and the positioning accuracy can be improved by $34 \%$.

Apart from the tropospheric products from the NWM model, researchers have conducted in-depth studies on the estimation and application of the troposphere based on PPP technology. Lu et al. [7] analyzed the tropospheric accuracy obtained by using real-time multi-GNSS orbit and clock products; the results showed that the accuracy of tropospheric products can be better than $1 \mathrm{~cm}$. Lu et al. [8] further adopted real-time ambiguity-fixed PPP for the tropospheric estimation, the root-mean-square (RMS) of the troposphere was $4.5 \mathrm{~mm}$ and $7.1 \mathrm{~mm}$, respectively. It was demonstrated that the multi-mode GNSS ambiguity-fixed technique can improve the accuracy of troposphere compared to the GPS signal positioning.

The obtained tropospheric products from PPP can be used for regional tropospheric modeling based on the GNSS reference station network. Hadas et al. [9] analyzed the accuracy of tropospheric delay from the regional GNSS network. The results showed that the standard deviation of the real-time tropospheric error was $8.8 \mathrm{~mm}$ and the RMS was $8.8 \mathrm{~mm}$; PPP verification showed that the convergence time was significantly shortened. Shi and De Oliveira et al. $[10,11]$ evaluated the use of the regional tropospheric model to enhance real-time PPP, and illustrated that the convergence time of PPP could be greatly shortened. Apart from the PPP application, high-precision tropospheric delay can be used for estimating precipitable water vapor (PWV). Lu et al. [12] compared the PWV time series with the very-long-baseline interferometry (VLBI) results, and proved that the accuracy of BeiDou PWV can still be used for real-time meteorology research.

Previous researches mainly focused on obtaining high-precision ZTDs and gradients for PPP processing. However, the accuracy of estimated tropospheric delay from PPP decreases when the atmosphere suffers from short-term dramatic change. Although the additional estimation of horizontal tropospheric gradient parameters can reflect the asymmetry of tropospheric horizontal changes, remaining unmodeled errors can be absorbed by carrier phase residuals. Yang et al. [13] proposed a tropospheric modeling method based on slant tropospheric delay (STD) to shorten PPP convergence. In their results, when the elevation angle was greater than 15 degrees, the accuracy of the STD based on NWM was only $3.5 \mathrm{~cm}$. If considering ground-based GNSS enhancement in STD estimation, the residual error after modeling can obviously be reduced. However, no further research was conducted to investigate the impact of STD on PPP.

In this study, precise STD models with consideration of the carrier phase residuals from PPP backward filtering were constructed. The effects of the STD model on PPP were investigated. 
This paper is organized as follows: after this introduction, Section 2 illustrates the zenith and slant tropospheric reconstruction strategies. Section 3 demonstrates the influence of high-precision STD on PPP performance. Section 4 presents the conclusions and perspectives.

\section{Zenith and Slant Troposphere Modelling Strategy}

Materials in PPP processing, precise satellite orbits, and clocks are usually corrected with external products, then the basic PPP processing model can be expressed as follows:

$$
\begin{gathered}
L_{r}^{s}=\rho_{r}^{s}+c\left(\delta t_{r}-\delta t^{s}\right)+m_{r}^{s} T_{r}-I_{r}^{s}+\lambda\left(N_{r}^{s}+B_{r}-B^{s}\right)+\ell_{L} \\
P_{r}^{s}=\rho_{r}^{s}+c\left(\delta t_{r}-\delta t^{s}\right)+m_{r}^{s} T_{r}+I_{r}^{s}+c\left(b_{r}-b^{s}\right)+\ell_{P}
\end{gathered},
$$

where $L$ and $P$ refer to un-differenced observations of the original carrier phase and pseudo-range, respectively; $\rho$ represents the geometric distance from the satellite to the receiver; $\delta t_{r}$ and $\delta t^{s}$ represent the difference between the receiver and the satellite clock, respectively; $T$ and $m$ represent ZTD and the corresponding mapping function, respectively; I represents the ionospheric delay in the slant direction; $N_{r}^{s}$ represents the ambiguity parameter of carrier phase observation, $B_{r}$ and $B^{s}$ represent carrier phase hardware delays for receiver and satellite, respectively; $b_{r}$ and $b^{s}$ denote code hardware delay of receiver and satellite, respectively; and $\ell_{L}$ and $\ell_{P}$ denote the unmodeled errors of carrier phase observations and pseudo-range observations respectively, and in which including the influence of multipath error, observation noise, and so on.

Zenith tropospheric delay reflects the vertical changes of the atmosphere, whereas the gradient parameters reflect the horizontal changes of the atmosphere. On this basis, ZTD and the gradient parameters can be used to construct STD, which can reflect the 3D distribution of atmosphere. The equation can be written as:

$$
\delta T=m_{H} Z H D+m_{W} Z W D+m_{G}\left[G_{N} \cos (A)+G_{E} \sin (A)\right],
$$

where $Z H D$ and $Z W D$ represent the dry and wet components of the tropospheric delay in the zenith direction, respectively; $G_{N}$ and $G_{E}$ represent the tropospheric gradient in the direction of horizontal east and north, respectively [14]; $m_{H}, m_{W}$, and $m_{G}$ represent the mapping function of the tropospheric dry delay, wet delay, and horizontal gradients, respectively.

During the PPP processing, ZTDs and associated gradients are estimated with proper stochastic models, which means that the estimates are constrained by the prior variance and its propagation value. Owing to the high variability of the water vapor distribution, or the lower weight of the satellite in low elevations, the estimated tropospheric parameters cannot reflect the true variations of atmosphere. Therefore, parts of the tropospheric delays can be absorbed by the carrier phase residuals. Based on this, we propose to construct the STD considering the residuals, which can be expressed as follows:

$$
S T D=\delta T+\text { res }
$$

where res represents the carrier phase observation residual.

Carrier phase observation residuals are combinations of the unmodeled errors, multipath errors, and observations noise. Figure 1 illustrates the carrier phase residual from forward filtering (SMT00) and backward filtering (SMT24). The posterior residual changes with the satellite elevations, and the backward filter can reduce the residual without affecting its distribution. However, the residuals can be affected by the systematical error, which is clearly observed from BDS residuals.

As the final satellite orbit and clock products are used for post-processing, the unmodeled errors in the residuals are mainly the unmodeled troposphere; satellite antenna phase center errors may also exist [15]. Therefore, the use of raw phase residuals from backward PPP filters to reconstruct the STD products can help improve the performance of post-processed PPP. 


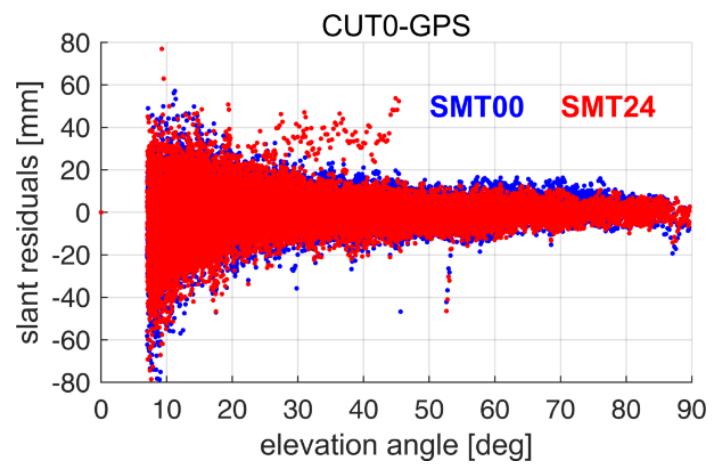

(a)

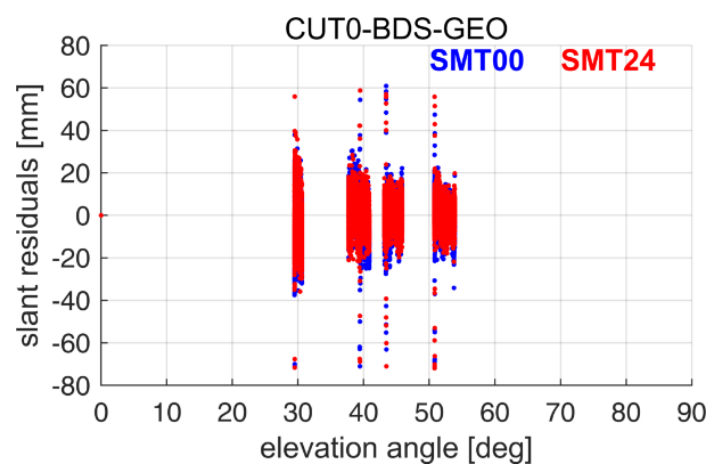

(c)

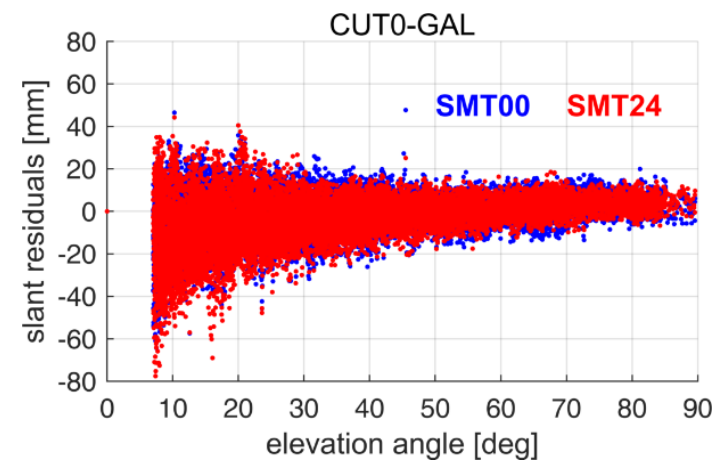

(b)

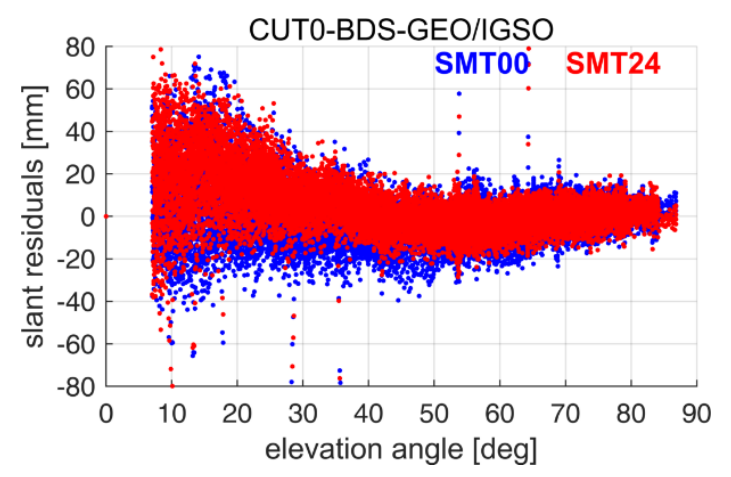

(d)

Figure 1. Carrier phase observation residual from CUT0 station by forward filtering (SMT00) and backward filtering (SMT24). There are four kinds of satellite systems: (a) CUT0-GPS. (b) CUT0-Galileo. (c) CUT0-BDS-GEO. (d) CUT0-BDS-GEO/IGSO.

\section{Experiments and Analysis}

To conduct the numerical experiment, the forward and backward filtering were used to process the observations based on the PPP method and obtain the precise STD and residuals. Then the results were saved as a SINEX_TRO 2.0 correction format, which supports conventional tropospheric parameters, including ZTD, ZWD, ZHD, carrier phase residuals, and multipath information. Finally, the correction files were introduced for simulating the real-time kinematic PPP process, and the effects of the troposphere on the PPP were comprehensively evaluated. Note that the specific processing strategies for the troposphere estimation are shown in Table 1.

Table 1. Processing strategies for the troposphere estimation.

\begin{tabular}{ll}
\hline \multicolumn{1}{c}{ Item } & \multicolumn{1}{c}{ Strategies } \\
\hline Estimator & Forward Kalman/backward smoothing \\
Satellite orbits & Fixed \\
Satellite clock offsets & Fixed \\
Observations & Carrier phase and pseudo-range observations \\
Observation weighting & Elevation-dependent weight \\
Elevation mask angle & Five degrees \\
& ZHD: Saastamoinen model \\
Zenith troposphere delay & ZWD: estimated with random-walk \\
& Mapping function: Global Mapping Function (GMF) \\
Troposphere gradient & Estimated, epoch-wise random-walk \\
\hline
\end{tabular}




\subsection{The Influence of STD on PPP Accuracy}

In order to evaluate the marginal effects of STD modeling on the convergence of PPP float solutions, observations from eight GNSS reference stations located in European region during 149 to 159 in 2013 were used. The distribution of the eight stations from the European Permanent Network (EPN) is presented in Figure 2. The corresponding ZTD, horizontal gradients, and precise STD products were calculated and applied for kinematic PPP. Four different positioning modes were considered, as illustrated in Table 2. Note that the external zenith tropospheric and horizontal gradient corrections were estimated with the G-Nut/Tefnut software with backward smoothing [16-18].

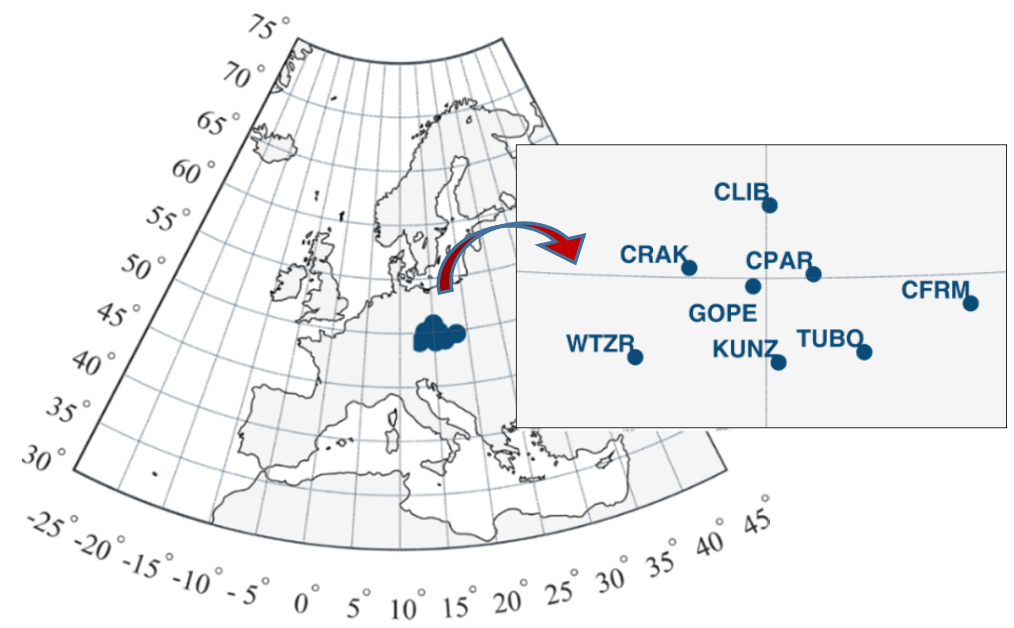

Figure 2. Distribution of stations.

Table 2. The positioning modes and their corresponding abbreviations.

\begin{tabular}{cc}
\hline Strategy & Positioning Modes \\
\hline est-ZTD & troposphere is modeled as a parameter \\
fix-ZTD & external zenith tropospheric correction products \\
fix-ZTDG & external zenith tropospheric and horizontal gradient correction products \\
fix-STD & STD correction products considering residuals \\
\hline
\end{tabular}

Figure 3 illustrates the statistical RMS on the N/E/U direction calculated by different tropospheric correction strategies. The precision of simulated real-time kinematic PPP was calculated after a given convergence period. There were eight stations lasting 11 days for observation during the experimental period, thus 88 PPP solutions were obtained in total. In general, the positioning accuracy on the north direction was better than that on the east and up directions. With the increase of convergence time, the accuracy of PPP was improved; however, minor accuracy improvement could be observed after $30 \mathrm{~min}$. Obvious precision improvement could be observed in the U direction when the "fix-ZTD" and "fix-ZTDG" strategies were used, whereas the effects on tropospheric gradient parameters were minor. The positioning accuracy in N/E/U reached $1.5 \mathrm{~cm} / 1.8 \mathrm{~cm} / 2.6 \mathrm{~cm}$ for the strategy "fix-ZTD", whereas the positioning accuracy in N/E/U direction was $0.5 \mathrm{~cm} / 1 \mathrm{~cm} / 1.2 \mathrm{~cm}$ for the "fix-STD" strategy. Precision improvements could be observed in all directions when adopting the "fix-STD" strategy, of which the improvement in the $\mathrm{U}$ direction was significant. 


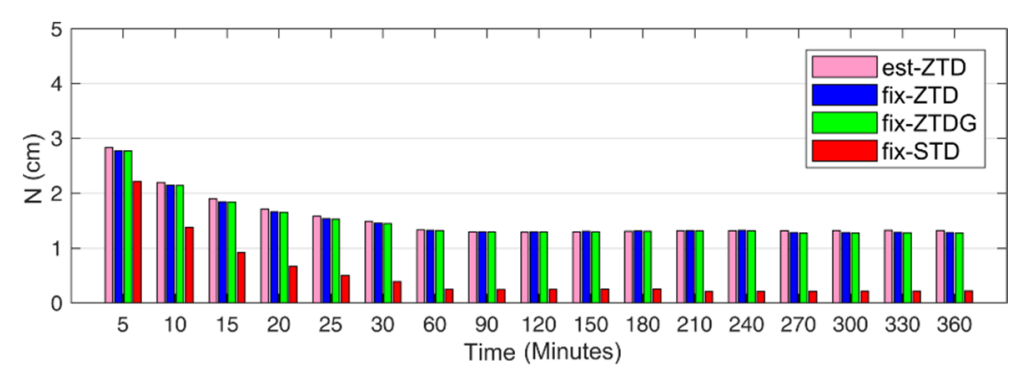

(a)

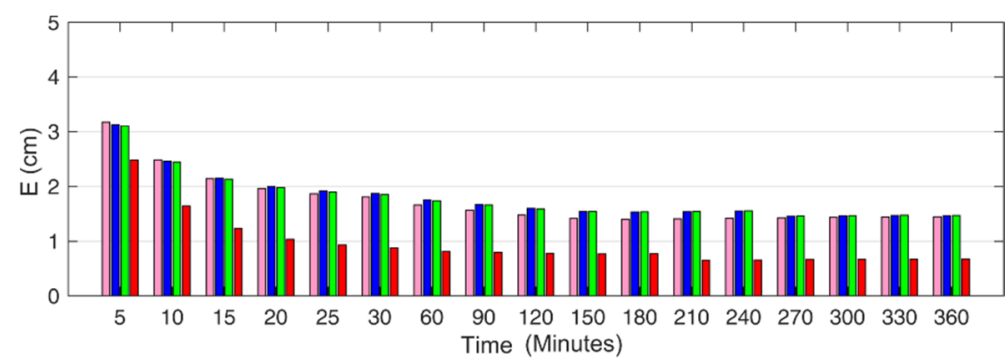

(b)

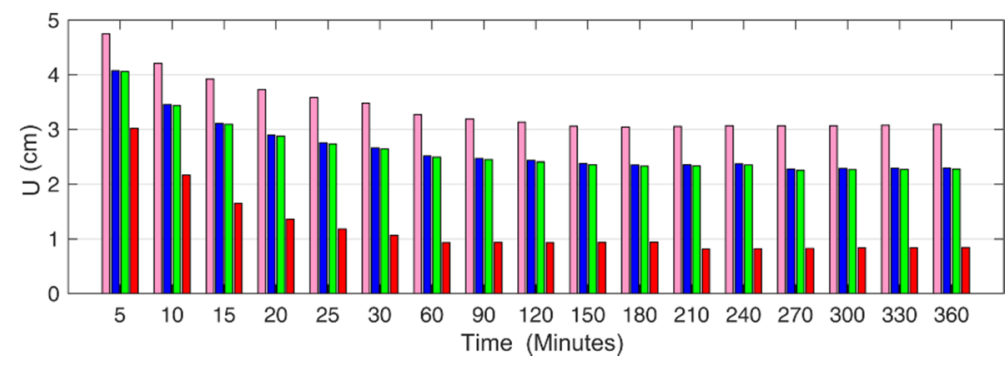

(c)

Figure 3. Coordinate precision statistical results after different convergence periods with four different tropospheric correction strategies. The $x$ axis represents the convergence time used for the precision statistics of PPP. (a) North. (b) East. (c) Up.

Figure 4 illustrates the positioning error series of the CFRM station which suffered large tropospheric variations. It was observed that the positioning accuracy in the east direction is significantly improved with the help of the horizontal gradient (the "fix-ZTDG" strategy) during the convergence period. In the case of the STD augmentation (the "fix-STD" strategy), the convergence and accuracy were further improved, which was obvious in the north and up directions. Since the observation noise was not eliminated in the STD modeling based on Equation (3), the observations in PPP processing were smoothed when applying the STD corrections. Therefore, that the positioning errors series for the "fix-STD" strategy is rather smooth. The results also demonstrated that the elimination of multipath and observations noise in the "fix-STD" strategy could improve the convergence and positioning accuracy. 


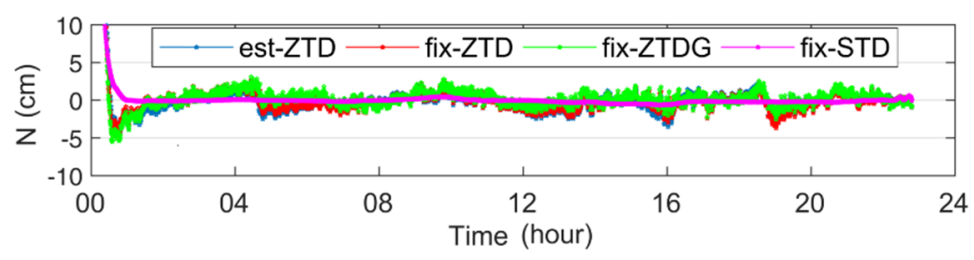

(a)

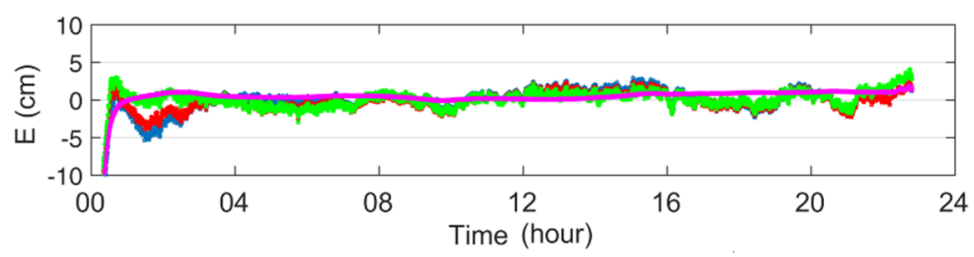

(b)

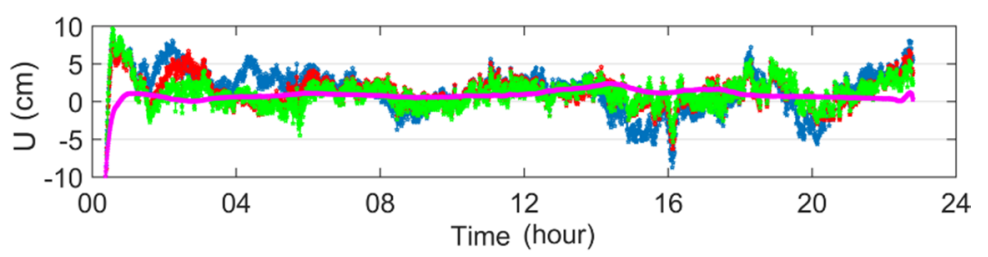

(c)

Figure 4. The positioning error series of the CFRM station (2013-05-20) calculated by different tropospheric correction strategies. (a) North. (b) East. (c) Up.

\subsection{The Influence of STD on the Parallel PPP Receiver}

It was the unmodeled tropospheric errors, rather than multipath or observational noise in STD models, that improved the PPP accuracy, therefore three parallel stations were selected for data processing. Figure 5 illustrates the location of the stations (CUAA, CUT0 and CUTC), among which CUTC and CUT0 used the same receiver. The ZTD difference for the three stations was ignorable due to their close location. The observation from CUT0 was used for STD modeling, then its ZTD and STD products were used to augment CUTC and CUAA for float and fixed PPP. Furthermore, the STD products calculated from the same receiver were also used for comparison.

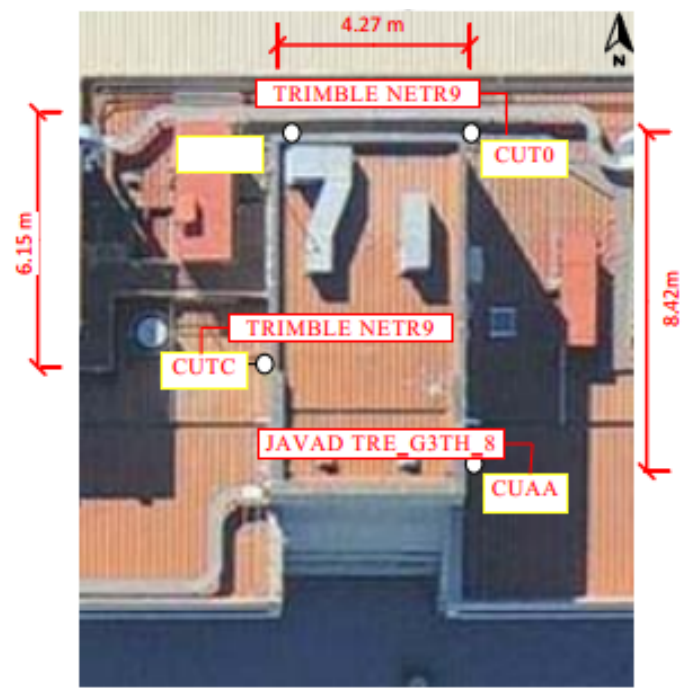

Figure 5. Station distribution and corresponding receivers. 
Figure 6 presents the positioning error series of the PPP float solution based on four different tropospheric corrections on Day of Year (DOY) 253 of 2017 for the CUTC station located in Curtin University. Minor positioning accuracy improvement was observed when we adopted ZTD corrections. In comparison, the positioning accuracy in the E direction around 20:00 and the U direction near 4:00 were significantly improved when adopting the STD correction of the external CUT0 station (the "slant (cut0)" strategy). The positioning error series were rather smooth for the "slant (cutc)" strategy when the same station STD products were used.

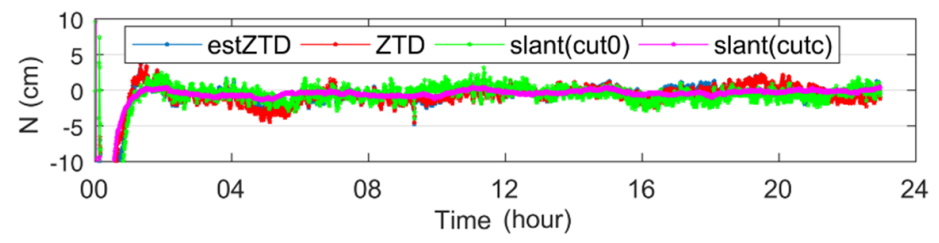

(a)

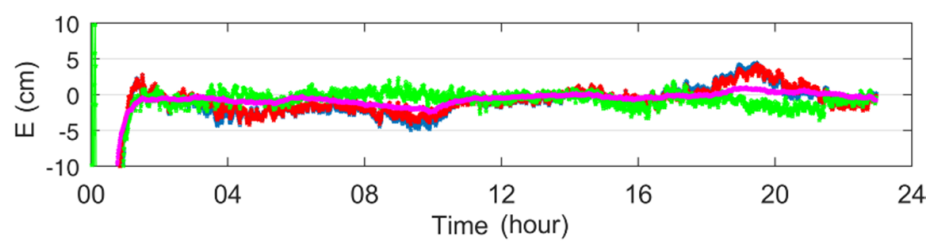

(b)

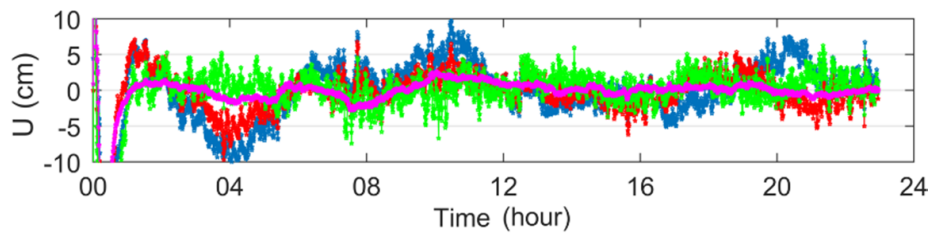

(c)

Figure 6. Positioning errors of float the precise point positioning (PPP) solutions of CUTC using different tropospheric corrections. (a) North. (b) South. (c) Up.

Table 3 presents the statistical RMS of the two stations (CUTC and CUAA) calculated by different tropospheric correction models. The "fix-STD" strategy represents PPP using the same station STD products. In comparison to the "fixZTD" strategy, the accuracy improvement for the "fix-STD(CUT0)" strategy was $14 \% / 28 \% / 31 \%$ in the N/E/U direction, respectively. The positioning accuracy could be further improved when the same station STD correction was used, and the positioning accuracy of the $\mathrm{N} / \mathrm{E} / \mathrm{U}$ direction was improved by $51 \% / 51 \% / 60 \%$ compared to the "fix-ZTD" strategy.

Table 3. RMS values of kinematic PPP using different modeling strategies.

\begin{tabular}{ccccccc}
\hline RMS/m & \multicolumn{3}{c}{ CUAA } & & CUTC \\
& North & East & Up & North & East & Up \\
\hline est-ZTD & 0.0107 & 0.0183 & 0.0351 & 0.0111 & 0.0195 & 0.0372 \\
fix-ZTD & 0.0106 & 0.0160 & 0.0222 & 0.0124 & 0.0174 & 0.0239 \\
fix-STD(CUT0) & 0.0091 & 0.0115 & 0.0174 & 0.0102 & 0.0116 & 0.0187 \\
fix-STD & 0.0052 & 0.0077 & 0.0074 & 0.0052 & 0.0089 & 0.0094 \\
\hline
\end{tabular}

As it is illustrated, the proposed STD correction could improve the PPP float accuracy compared to the traditional ZTD correction. We then analyzed the influence of STD correction on the PPP ambiguity-fixed solutions. The positioning error series of the fixed PPP solutions using different 
correction strategies are given in Figure 7. The estimated parameters are re-initialized every $4 \mathrm{~h}$ in the experiment. It can be seen that the ambiguity-fixed PPP accuracy was not decreased using STD correction products. Moreover, the fixing rate used the "fixSTD" strategy in the E direction was improved during 16-20 h. The statistical results demonstrate the accuracy for the "fixSTD" strategy could improve by $14 \% / 28 \% / 21 \%$ in the N/E/U direction, and the fixing rate of PPP ambiguity was increased by $30 \%$.

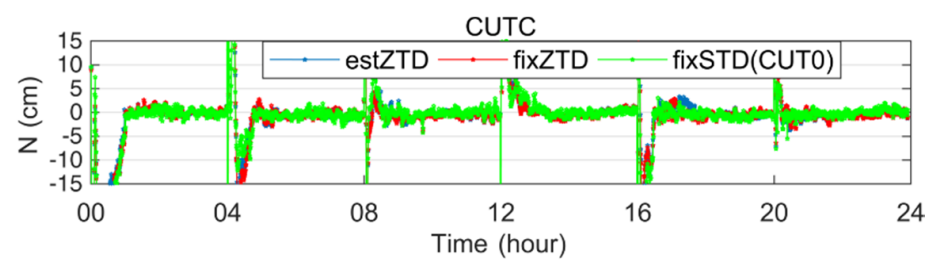

(a)

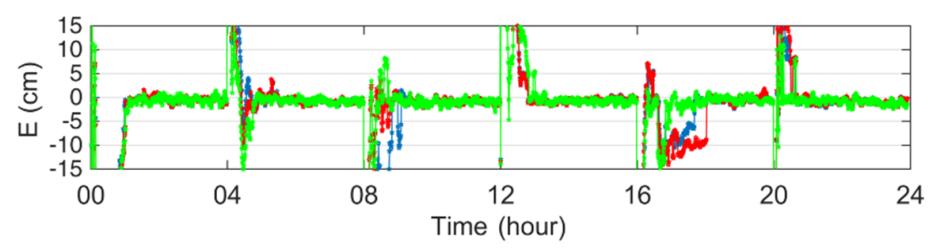

(b)

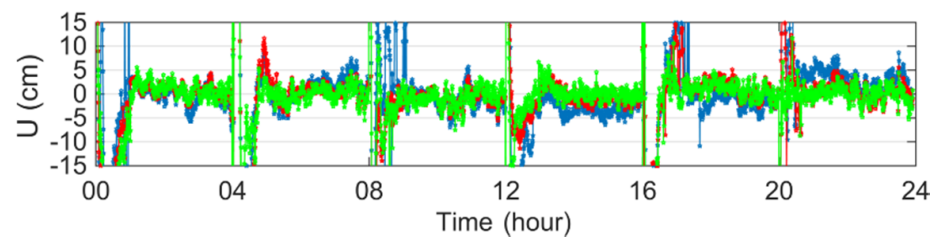

(c)

Figure 7. The improvement of different troposphere modeling strategies to PPP ambiguity-fixed solutions. (a) North. (b) South. (c) Up.

The comparisons of PPP ambiguity-float and fixed solutions obtained by different STD models are given in Figure 8. It was observed that the ambiguity-fixed PPP could improve the positioning accuracy, especially in the E direction. The "fixSTD-fix" strategy suffered from large noise due to the fact that the unmodeled errors and observations noise in the STD modeling were not eliminated. The influence of observation noise was eliminated for the "fixSTD-fix (Self)" strategy, thus the obtained positioning coordinate sequence was rather smooth. Moreover, the positioning convergence time could be shortened, and the accuracy of PPP ambiguity-float solutions and the fixing rate of ambiguity resolution were further improved.

The BDS PPP suffered longer convergence time compared to GPS due to its less-accurate orbit and clock solutions. Therefore, the performance of different troposphere corrections on BDS PPP were further evaluated. As the phase biased products for BDS ambiguity-fixed solutions were not available, only ambiguity-float solutions using different tropospheric correction strategies are shown in Figure 9. It was observed that the positioning accuracy with STD constraints were significantly improved compared to the ZTD constraints. The positioning errors in the $\mathrm{E}$ and $\mathrm{U}$ directions obtained by the "tropZTD" strategy around 19:00 were significant, while the "tropSTD" strategy could decrease the positioning errors. The results indicated that the unmodeled residuals in the STD improved the positioning accuracy, and that the elimination of multipath error and observation noise in STD modeling could further smooth the results. However, the contribution of the different strategies to the 
convergence was not obvious, which demonstrated that the orbit and clock accuracy, especially the poor GEO orbits, were the primary reasons limiting the convergence of BDS PPP.

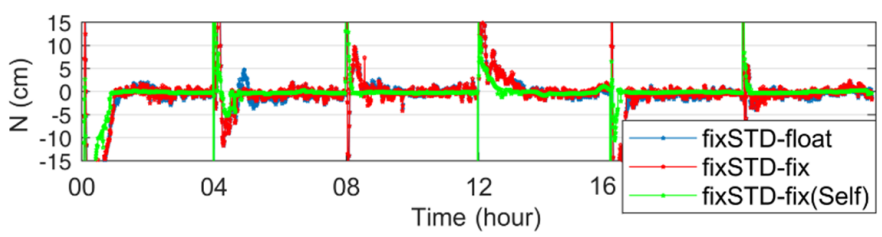

(a)

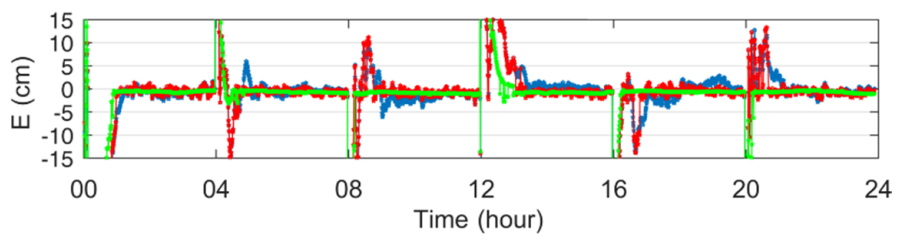

(b)

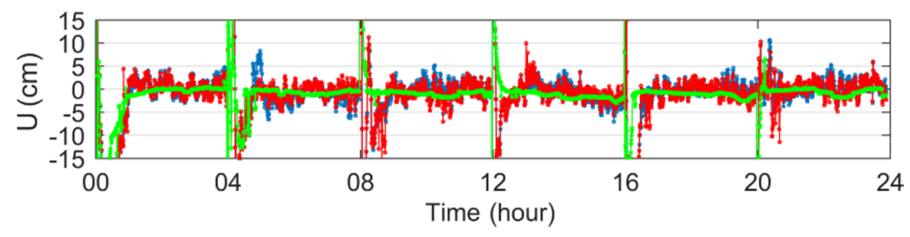

(c)

Figure 8. The PPP ambiguity-float solutions and fixed solutions of CUTC obtained by different slant tropospheric delay (STD) models. (a) North. (b) South. (c) Up.

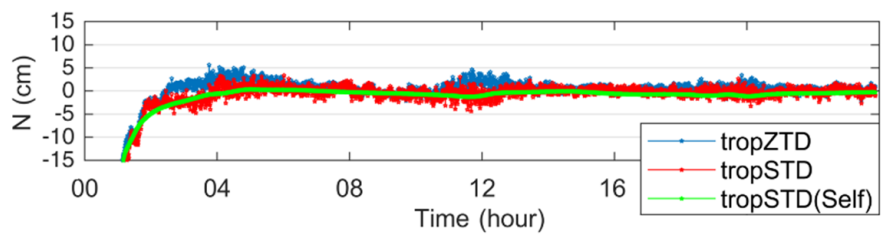

(a)

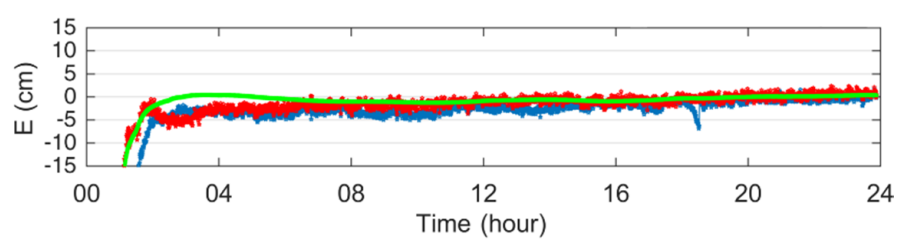

(b)

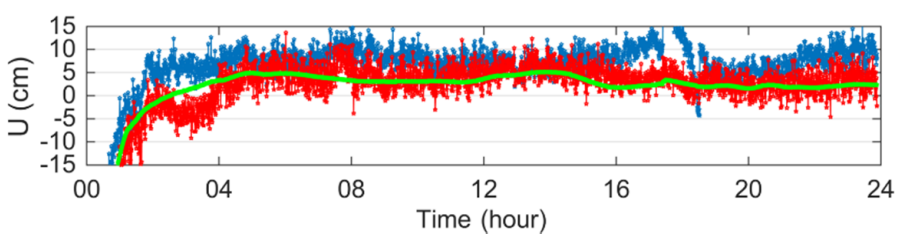

(c)

Figure 9. The PPP ambiguity-float solutions of CUTC using different constraint strategies. (a) North. (b) South. (c) Up. 


\section{Conclusions}

To improve the accuracy and reliability of PPP positioning, the STD-augmented PPP model was proposed and validated. Different from the traditional method where the STD is obtained by ZTD and horizontal gradients, the carrier phase residuals obtained from backward PPP filter is considered to improve the precision of STD. The precise STD model is then used to augment the PPPfloat and fixed solutions.

First, the marginal effects of STD on PPP accuracy were investigated based on the observations from European GNSS stations. Results indicated that the ZTD and STD modeling based on ZTD + gradients mainly improved the vertical coordinate accuracy, whereas the consideration of the carrier phase residuals for STD modeling could improve the three-dimensional accuracy to $0.5 \mathrm{~cm} / 1 \mathrm{~cm} / 1.2 \mathrm{~cm}$ in the N/E/U component.

Then, three parallel GNSS receivers were selected to investigate the impacts of external STD corrections on ambiguity-float and fixed PPP. Results indicated that external STD correction could improve the RMS of positioning errors in the N/E/U direction by $14 \% / 28 \% / 31 \%$ compared to ZTD augmentation. In comparison, $51 \% / 51 \% / 60 \%$ improvement could be observed when the same station STD corrections are used. Similar improvements were also observed for the PPP fixed solutions, and the ambiguity-fixing rate could be improved with the STD correction. In addition, BDS observations were used to validate the improvement of STD augmentation. Results indicated that the positioning accuracy of STD augmentation could be improved compared with the ZTD strategy, and the positioning series could be smoothed if the same station STD correction was used.

Overall, experimental results indicated that the STD corrections from the same station and the external station could both improve the PPP accuracy, which validated the proposed method. Furthermore, it was the unmodeled tropospheric errors, rather than the multipath and observation noise in STD modeling, that improved PPP.

Author Contributions: Conceptualization, C.X.; Formal analysis, L.Y.; Software, L.Z.; Writing—original draft, C.X., L.Y. and L.Z.; Writing-review \& editing, C.X.

Funding: This research received no external funding.

Acknowledgments: We appreciate the Curtin GNSS Research Centre and IGS for providing the observation data.

Conflicts of Interest: The authors declare no conflict of interest.

\section{References}

1. Shi, J.; Gao, Y. A troposphere constraint method to improve PPP ambiguity-resolved height solution. J. Navig. 2014, 67, 249-262. [CrossRef]

2. Jiang, P. The Study of Retrieving 2D/3D Water Vapor Distribution Using Ground-Based GNSS Meteorology. Ph.D. Thesis, Wuhan University, Wuhan, China, 2014.

3. Dousa, J.; Vaclavovic, P. Real-time zenith tropospheric delays in support of numerical weather prediction applications. Adv. Space Res. 2014, 53, 1347-1358. [CrossRef]

4. Lu, C.; Zus, F.; Ge, M.; Heinkelmann, R.; Dick, G.; Wickert, J.; Schuh, H. Tropospheric delay parameters from numerical weather models for multi-GNSS precise positioning. Atmos. Meas. Tech. 2016, 9, 5965-5973. [CrossRef]

5. Lu, C.; Li, X.; Zus, F.; Heinkelmann, R.; Dick, G.; Ge, M.; Wickert, J.; Schuh, H. Improving BeiDou real-time precise point positioning with numerical weather models. J. Geod. 2017, 91, 1019-1029. [CrossRef]

6. Zhu, K.; Zhao, L.; Wang, W.; Zhang, S.; Liu, R.; Wang, J. Augment BeiDou real-time precise point positioning using ECMWF data. Earth Planets Space 2018, 70, 112. [CrossRef]

7. Lu, C.; Chen, X.; Liu, G.; Dick, G.; Ge, M.; Wickert, J.; Jiang, X.; Zheng, K.; Schuh, H. Real-time tropospheric delays retrieved from multi-GNSS observations and IGS real-time product streams. Remote Sens. 2017, 9, 1317. [CrossRef] 
8. Lu, C.; Li, X.; Cheng, J.; Dick, G.; Ge, M.; Wickert, J.; Schuh, H. Real-time tropospheric delay retrieval from multi-GNSS PPP ambiguity resolution: Validation with final troposphere products and a numerical weather model. Remote Sens. 2018, 10, 481. [CrossRef]

9. Hadas, T.; Kaplon, J.; Bosy, J.; Sierny, J.; Wilgan, K. Near-real-time regional troposphere models for the GNSS precise point positioning technique. Meas. Sci. Technol. 2013, 24, 055003. [CrossRef]

10. Shi, J.; Xu, C.; Guo, J.; Guo, J.; Gao, Y. Local troposphere augmentation for real-time precise point positioning. Earth Planets Space 2014, 66, 30. [CrossRef]

11. De Oliveira, P.; Morel, L.; Fund, F.; Legros, R.; Monico, J.F.G.; Durand, S.; Durand, F. Modeling tropospheric wet delays with dense and sparse network configurations for PPP-RTK. GPS Solut. 2017, 21, 237-250. [CrossRef]

12. Lu, C.; Li, X.; Nilsson, T.; Ning, T.; Heinkelmann, R.; Ge, M.; Glaser, S.; Schuh, H. Real-time retrieval of precipitable water vapor from GPS and BeiDou observations. J. Geod. 2015, 89, 843-856. [CrossRef]

13. Yang, L.; Hill, C.; Moore, T. Numerical weather modeling-based slant tropospheric delay estimation and its enhancement by GNSS data. Geo-Spat. Inf. Sci. 2013, 16, 186-200. [CrossRef]

14. Bar-Sever, Y.E.; Kroger, P.M.; Borjesson, J.A. Estimating horizontal gradients of tropospheric path delay with a single GPS receiver. J. Geophys. Res. Solid Earth 1998, 103, 5019-5035. [CrossRef]

15. Kačmařík, M.; Douša, J.; Dick, G.; Zus, G.; Brenot, H.; Möller, G.; Pottiaux, E.; Kapłon, J.; Hordyniec, P.; Václavovic, P.; et al. Inter-technique validation of tropospheric slant total delays. Atmos. Meas. Tech. 2017, 10, 2183-2208. [CrossRef]

16. Douša, J.; Václavovic, P.; Zhao, L.; Kačmařík, M. New adaptable all-in-one strategy for estimating advanced tropospheric parameters and using real-time orbits and clocks. Remote Sens. 2018, 10, 232. [CrossRef]

17. Kacmarik, M.; Dousa, J.; Zus, F.; Vaclavovic, P.; Balidakis, K.; Dick, G.; Wickert, J. Sensitivity of GNSS tropospheric gradients to processing options. Ann. Geophys. 2019, 37, 429-446. [CrossRef]

18. Vaclavovic, P.; Dousa, J. Backward smoothing for precise GNSS applications. Adv. Space Res. 2015, 56, 1627-1634. [CrossRef]

(C) 2019 by the authors. Licensee MDPI, Basel, Switzerland. This article is an open access article distributed under the terms and conditions of the Creative Commons Attribution (CC BY) license (http://creativecommons.org/licenses/by/4.0/). 